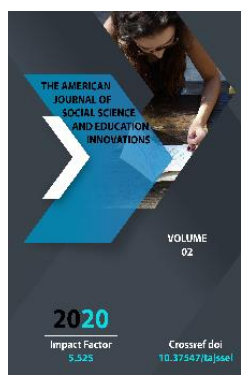

Copyright: Original content from this work may be used under the terms of the creative commons attributes 4.0 licence.

\section{Development Of Personality Traits In School-Age Children}

\author{
Bobomurodova Tojihol Norbadalovna \\ Teachers Of The Denau Institute Of Entrepreneurship And Pedagogy, Uzbekistan \\ Toshboyva Farida Abduxalilova \\ Teachers Of The Denau Institute Of Entrepreneurship And Pedagogy, Uzbekistan \\ Ergasheva Oyjamol Jahongirovna \\ Teachers Of The Denau Institute Of Entrepreneurship And Pedagogy, Uzbekistan \\ Turdiyeva Izzatoy Sodiqovna \\ Teachers Of The Denau Institute Of Entrepreneurship And Pedagogy, Uzbekistan
}

\title{
ABSTRACT
}

In school-age children, personality traits and ways of its formation are mentioned. The socialization of the child, the negative features of his physical and mental development and the factors that cause it are studied. The principle of the person is stated that each stage of human development is an individual and social value.

\section{KEYWORDS}

Person, social education, ethics, vocational rehabilitation, etiquette, social factors.

\section{INTRODUCTION}

Formation of personality traits in school-age children. One of the most important tasks today is to develop small school-age students into well-rounded, well-mannered and wellmannered people. Because it stems from the issue of increasing the effectiveness of education and upbringing in the process of restoring national values and spirit. In order to successfully carry out the task facing psychologists in the study of this problem, it is necessary to take into account the individual characteristics of students in order to properly 
organize primary education on a scientific basis. Learning activities provide an opportunity for a small school-age student not only to develop a high level of cognitive processes, but also to develop personal characteristics.

In addition to leading learning activities, play, communication, and work also have a direct impact on a student's personal development. Based on these activities, personal characteristics related to the motives for success begin to take shape. While learning activities satisfy students 'need for academic success, they also provide opportunities for them to gain a place among their peers. It is possible for a child to study well in order to achieve this position. Children this age constantly compare their success with that of their peers. It is important for them to always be first.

In children of this age, the motivation to enter the competition is a constant need, which gives them a strong emotional tension. These traits actually begin in kindergarten and are most pronounced in elementary school and adolescence. Children's self-esteem, based on the assessments and opinions of adults about it, largely depends on the teacher's assessment and success in various activities. The characteristics of school-age children, such as confidence, openness, sensitivity to external influences, and obedience, provide a good opportunity to nurture them as individuals. This period is a period of emergence and strengthening of the basic personality traits that determine the success of the child in various activities. During this period, as the motives for success are formed, such qualities as diligence and independence are strengthened. Hard work is only possible if the child enjoys what he is doing.
The development of a child's independence depends largely on the adult. If the child is overconfident, obedient, open, then gradually develops the qualities of obedience and dependence. However, encouraging a child to become independent over time can also lead to the development of some negative traits in him. Because he learns life experiences by imitating someone.

To build independence, it is important to give the child more independent work and confidence. It is also necessary to create a social psychological environment in which the child is assigned to perform an independent, social task, in the process of which the child feels like a leader of peers, adults and other people. It is this feeling that motivates the child to be independent. A school-age child understands his or her individual characteristics. The child's self-awareness also develops and strengthens rapidly. During this period, children begin to pay more attention to their names and try to be positively received by their peers and those around them. The way a child evaluates his or her appearance and body shape is also important for self-awareness.

This means that the educator plays a special role in shaping the child's personality. In particular, it requires responsibility for the formation of each child as an individual. In this regard, the educator should set an example for children in everyday life, in games, activities, joint work, interaction with them, to study the child in all respects, to know his personality, abilities, It is necessary to show pedagogical decency, to be able to objectively assess the behavior of children, the results of their work and to provide them with timely assistance, as well as to be interested in their marital status. 
Our thinkers have expressed many opinions about the educator and his personality. In this regard, Navoi's "Human beauty is knowledge and etiquette. Suffice it to recall his wise words: "Only teachers who instill these in their students are true teachers."

Parents have a great influence on the development of habits in children from one to seven years of age. Therefore, the work of a social educator consists primarily of working with the child's family (with his parents) and the child himself. At this age, a child's desire to drink alcohol is often imitated by an adult. If during this period the child develops bad habits, including those who prefer to consume alcohol, then by the age of 14 they are fully formed. It will be very difficult to get rid of these bad habits.

Working with parents is done in two ways: Creating a culture of relationships between family members. Psychological guidelines against alcoholism can only be established when there is a decent family relationship. creating a healthy living environment for a child involves creating a negative attitude towards alcohol and people who consume alcohol, morally condemning the situation. The following goals are achieved when working with a child: A child's socialization can be complicated by negative features of his or her physical and mental development. The development of a child's social norms and values can go in the wrong direction due to the negative impact of the environment. As a result, the child is excluded from normal social relationships and needs special care. The process of socialization of such children is carried out primarily through upbringing.

In general, the task of teachers and professionals is to focus on the social adaptation of a child in a difficult situation, that is, to actively adapt to the laws and norms adopted in society or to eliminate the consequences of negative factors. Education as a social institution has certain functions in social life. General objectives of education:

- Creation of conditions for targeted development of community members and meeting a number of needs that can be met by them in the educational process;

- Training of personnel who can operate and develop in society;

- Ensuring the sustainability of social life through the transmission of culture to future generations;

- Social and spiritual development of community members;

- Adaptation of community members to changing social situations;

- Ensuring the unity of aspirations and goals of members of society and the harmony of interests of social, professional, ethnic and religious groups. Principles of education are the rules of education, on the basis of which theories are developed and methods, forms and means of teaching are implemented.

The principle of humanization of education.

In social pedagogy, the principle of humanity means that the educator treats the pupil as a responsible and independent subject of his own development. The implementation of the principle of humanization of education has a positive impact on the formation of children, adolescents and young people.

As a result of the implementation of this principle:

- How antisocial is a person who is the object of socialization? 
accepting positive norms and values, not normative ones

- Identifies;

- Education has the ability to create the conditions for a person to feel as a subject of socialization, to show his subjectivity and to develop;

- Education creates conditions for a person to develop a level of social adaptation.

- Education can prevent a person from being put at risk at a certain age, i.e. it reduces the chances of a person becoming a victim of socialization.

The practical application of the principle of humanization of education has a positive effect on the development of reflection and self-regulation in the pupil, the formation of his relationship with the world, the formation of a person who is a carrier of democratic and humanistic relations in society.

The principle of culturalization of education

The modern definition of the principle of harmony of education with nature, defined by the German pedagogue Friedrich Disterverg in the XIX century, is based on the universal values of educational culture and is built in accordance with the values and norms of one or another national culture.

The principle of variability of education. The variability of social upbringing in modern societies is determined by the diversity of individual needs.

The principle of educational diversity is based on universal human values at the regional, municipal and local levels, taking into account ethnic characteristics, as well as regional municipal and local conditions, to create conditions for spiritual and moral education in educational institutions on the basis of human, youth, differential and individual approaches.

In order to implement the principle of variability of social education, it is necessary to:

- Establishment of various educational institutions to meet the interests and needs of the individual, the needs of different age, socio-professional, ethnic groups, the needs of regional and regional organizations;

- Organization of regional and municipal systems of social education; organization of local educational systems.

Implementation of the principle of variability of social education:

- To form a person's ability to solve various life and personal problems;

- Creates conditions for people to expand their opportunities to choose their own development strategies, life and career paths.

The principle of the collective nature of social education. The description of the microcosm of socialization suggests that the socialization of children and adolescents occurs in their interactions with peers and adults (family, neighbors, microcommunity, educational institutions). Social upbringing takes place in communities as part of a relatively socially controlled socialization, on the one hand, and on the other hand, children and adolescents are brought into community life as an objective necessity. 
The idea that the community is the most important means of education dates back to antiquity, and from the 19th century onwards, educators have been working on its theoretical foundations. According to the modern definition of the principle of community, social education takes place in different communities, allowing a person to gain life experience, and the experience of adapting to society in general.

\section{The principle of centralization of education to personal development}

According to this principle, the strategy and goal of social education is to help the younger generation, adolescents to become fullfledged human beings. The process of social upbringing, educational institutions can only be a means of personal development.

Development can be seen as the process by which a person solves three types of tasks (natural-cultural, socio-cultural, sociopsychological). These tasks can be used to identify aspects of personal development that need to be centralized in social education in educational institutions.

The need to solve natural and cultural problems of human development

- Physical (natural);

- Helps to develop sexually (physiologically).

- The need to solve socio-cultural problems is one of the following:

- Intellectual;

- Creates the basis for social development.

The possibilities and place of these aspects of social education vary, but in each of these aspects it has an additional place in relation to the factors of socialization. In addition, the opportunities vary depending on the type of educational institution.

\section{The principle of joint action of social education:}

According to the principle of interaction of social education, the spiritual and educational development of children, adolescents and adolescents is carried out in the process of exchange of values (mental, social) between the educator and the pupil. These are:

- World cultures and values developed in a particular society;

- Values inherent in the subjects of social education;

- Values specific to a particular member of the educational institution.

The principle of incompleteness of education.

This principle stems from the hereditary nature of socialization. The principle of incompleteness of education considers each stage of human development as an individual and social value. According to this principle, every child and teenager will always have something unfinished and unfinished. According to the principle of incomplete upbringing, upbringing should be organized in such a way that at every age a person should be "reshaped".

Although the period of primary school age is not a period of decisive shifts in personality development, in our opinion, such a period is the period of adolescence, but in this period the formation of personality is of great importance. As mentioned above, school entry is a turning point in a child's life. New relationships are formed with adults (teachers) and peers (classmates). The child joins the whole community system (school, class). 
Adding a new type of activity that places a number of serious demands on the teacher forces the student to organize their life strictly and follow rules and regimes. All of this has a profound impact on the reality around us, other people, the community, education, and the responsibilities associated with it, and has a decisive impact on shaping and strengthening a new system of relationships. expands the range of interests, determines the development of skills.

The acquisition of moral norms and rules of conduct lays the foundation for moral behavior in the early school years, the acquisition of moral norms and rules of conduct. The social orientation of the individual begins to find content. The moral consciousness of small school-age students undergoes significant changes during the period from 1st grade to 4th grade. By the end of this age, the knowledge and perceptions of ethical knowledge will be significantly enriched, more understood, will have different concepts, will be generalized. - based on specific instructions and explanations of mothers. Grade 3-4 students, on the other hand, try to analyze other people's experiences based on their own behavioral experiences and adult guidelines in their ethical judgments.

It is important to watch children's films when reading such literature. Ethical behavior finds a similar content. Children ages 7-8 do many of these positive ethical activities from the beginning on their own initiative, without waiting for direct instruction from adults, including the teacher, to be positive.

Character. Behavioral behaviors in primary school-age children are not always stable and stable forms of behavior in which relationships take place. In small school-age children, the character begins to find serious content. The behavior of young school-age children can vary with their instability and inconsistency.

By the end of elementary school, boys, especially girls, begin to pay more attention to their facial features. In the process of their learning, they develop the ability to coordinate their own behavior and activities, to develop the ability to consciously agree, to develop an interest in self-organization and the process of learning. help you make a decision. The motivation of the student's behavior also changes. The main motives are the opinions of friends and the team. Moral feelings and willpower are formed. At the age of 5-6 years, the characteristics of the child develop and strengthen over the years.

By the beginning of adolescence, many personal qualities can be formed. Children's individuality is also reflected in their cognitive processes. During this time, children's knowledge expands and deepens, and their skills and abilities improve. In grades 3-4, the general and specific abilities of some students are highlighted. At a young school age, the motivation to succeed, which is extremely important for life, is strengthened, which in itself leads to the rapid development of other abilities. Because children of this age are fully developed in attention, comprehension, memory, thinking, and speech, adult-style teaching methods can also be used.

It is well-known that in order to set an example in the formation of a student's personality, it is necessary to structure their character traits and personal characteristics in accordance with the purpose. It allows for an individual relationship with a subject that is taken separately after each student has been individually identified. The personal qualities of 
an 8-10 year old child are unstable, his worldview is simple, and he is quick to give in to external impressions. As the great scholars have pointed out, a person's character is formed, above all, in the early knowledge of his life, and the quality manifested in this quality is firmly established and becomes the firm nature of the child. In the second nature of the child, all the responsibility for the formation of positive emotions, personality traits, the formation of high human qualities in them falls on the primary school teacher.

Children's emotions change depending on how the learning process is organized. In this way, students of primary school age are able to control their emotions and achieve emotional stability. By this time, they are in control of their emotions. Because they are calmer, deeper, and more balanced than preschoolers and teens. They are characterized by long-term positive emotions, cheerfulness, mood.

When children first come to school, a new system of relationships and a circle of communication is formed. From the first days of school, students do not pay attention to each other, are not interested in the achievements of their peers, and rarely communicate. But over time, students gradually begin to observe each other, feel the need for communication, and become friends with their peers. and by the end of primary education, students begin to need a special place among their peers. Interest in the educational achievements of their peers begins to grow, and the desire to stand out from them becomes the main motive of behavior. The formation of their personal qualities also depends on cooperation in educational activities.
In our hypothesis of our research, we planned to study the extent to which their selfassessment influenced the formation of small school-age grandparents as individuals. To do this, we conducted Dembo Rubinstein's "SelfAssessment" method in the 2nd and 3rd grade students of secondary school No. 6 in Piskent district (30 in total). This methodology is based on the direct assessment (expression on scales) of a number of personal qualities of schoolchildren, such as health, character, intelligence, and so on. examinees are asked to indicate how well they have developed these qualities (self-assessment) and how well they have developed these qualities, and to indicate their satisfaction (level of suitability) with certain signs on the vertical lines. Each examinee is given a form with instructions and tasks on the method.

\section{Social factors:}

Biological inheritance alone is not enough to grow into a human being. Man becomes a person only in the process of socialization, that is, in communication, in interaction with other people. Spiritual, social, and spiritual development cannot take place outside of human society. This idea is reinforced by the well-known fact that human beings grow up among animals.

Socialization is a multifaceted process that takes place throughout a person's life. He is extremely active, especially in childhood and adolescence. Because it is in childhood that the basic social norms are learned.

Sociality plays an important role in a child's socialization. The child gradually adapts to this social environment. If a child develops mainly in the family after birth, his further development will take place in new and new environments - preschools, schools, out-of- 
school educational institutions, various entertainment venues. As you grow older, the "territory" of the social environment expands. The more environments a child learns, the more space he or she will try to occupy. The child always tries to find an environment that is comfortable for him, understands him well and treats him with respect. So he moves from one environment to another. The environment is important for the formation of the child, for his social experience, for the process of socialization. The environment is not just a street, a house, or anything else that a person needs to know just enough to get in and feel comfortable. Perhaps the environment is also a community of people characterized by specific systems and rules of interaction. Therefore, man innovates, influences and changes the environment to a certain extent, and in turn, the environment puts its own demands on man. He can both accept and deny man, his actions. The attitude of the environment towards a person can be determined by how well a person behaves and meets his requirements. A person's behavior is determined by his or her place in society.

A child's mastery of behavioral mechanisms allows him to successfully adapt to social relationships. social adaptation is the adaptation of an individual to the conditions of the social environment.

Social adaptation is a condition and result of a child's successful socialization. This is done in three main areas: activity, behavior, and awareness. In the field of activity, the child expands the types of activities, acquires the necessary forms and means of activity, expands the scope of interaction in the field of behavior, deepens its content, learns the norms of behavior accepted in society. In the realm of consciousness, there is the formation of the image of the "I", the realization of one's social affiliation and social status. All of these processes are regulated by upbringing.

In recent years, the relationship between socialization and education has been widely discussed in pedagogy and other disciplines. Some authors are trying to replace upbringing with socialization. Others see parenting as part of a child's socialization. Some scholars, on the other hand, understand socialization as civic and moral education. The fourth group of scientists considers the socialization of the individual as the main goal of education. It is a well-known fact that proper upbringing is one of the main factors in a child's socialization.

Upbringing, which is an integral part of the influence of social factors on a person, has its own characteristics.

In particular, the impact of parenting on a child's development changes over time. In other words, the younger the child, the more the upbringing affects his or her development. Over time, that is likely to change.

Deviations in a child's social development are caused by poor families. There are ways of family interaction that lead to the formation of juvenile antisocial behavior:

1. Disharmonious method of educational and family relations. This method is based on the child's wishes on the one hand, and on the child's conflict situations on the other or the existence of two different moral views in the family: educational situations that are different for the family and completely different for society. An unstable, conflict-ridden approach has an educational impact on unhappy families, resulting in divorce, parental and children living apart for long periods of time. 
The associative pattern of relationships occurs in poorly organized families, with vices such as constant alcohol consumption, drug addiction, immorality, parental criminal behavior, “domestic violence," and violence.

Violent treatment (insult, humiliation) refers to the many acts of harm caused by people who take care of a child. These actions include physical, emotional, and sexual abuse of a child, as well as repeated inappropriate punishments. Violent relationships can occur at home, in the family, on the street, at school, in orphanages, hospitals and other children's facilities. Children in such situations do not feel safe. This feeling is very important for their normal development.

This makes the child think that he is bad, that he is not needed, that he is not loved. Any kind of abusive relationship can be very harmful to a child's health. It threatens his life and social resilience.

Socio-economic factors contribute to social inequality. It divides society into rich and poor, the most important for society is poverty, unemployment. Restrictions on socially accepted honest (legal) methods of obtaining wages lead to inflation and social unrest. Moral factors. While the decline of morality in modern society on the one hand, it leads to the emergence of valuable, first of all, the deterioration of moral behavior, on the other hand, in deviant behavior in the neutral attitudes of society.

Basic approaches to the prevention of deviant behavior in children. The continuous growth of various deviant behaviors has set the task of society to find forms and methods of work with children and young people who deviate from social norms.
Therefore, the two main technologies of working with children with deviant and delinquent behavior, preventive and rehabilitative work, are widespread in science and practice.

Prevention is a set of socio-medical and organizational-educational measures of the state, aimed at preventing, eliminating or neutralizing the conditions that are the main cause of various manifestations of social and behavioral disorders in children's behavior. . There are several approaches to preventing deviant behavior in children.

The unity and interdependence of a person's qualities form his psychological structure, his dynamic structure. What is the purpose of studying a person, his structure? The purpose of studying a person, his structure, is to identify the individual characteristics that distinguish him from other people. This leads to the separation and study of different "personal languages" on the basis of psychological research and theoretical work in this area. Creating a typology of individuals requires finding individual psychological characteristics in different people and grouping them into specific categories based on their common traits and characteristics.

Among the theories, there are two parts of the personality, the two factors that distinguish them. They distinguish between two factors in the structure of personality, biological and social. It is well-known that a person, being a social being, retains the features of his natural, biological structure. But the two factors cannot be contrasted, they form a unity, they emerge together.

A person is formed under the influence of external living conditions, but two children raised in the same conditions are two different 
things. Because their natural abilities are different. This means that in addition to social factors, biological factors also affect the formation of personality. These two things are inextricably linked.

1. Informational approach. This approach is based on the fact that juveniles (who do not know these social norms) do not know the origin of moral deviations from the social norm, so it is important to inform minors about their observance of these norms, their rights and responsibilities. consists of. In order to meet the requirements of the state and society, it is necessary to provide information about the social norms adopted in accordance with the age of children. Mass media (press, radio, television), cinema, theater, fiction and other works of art can be used to do this.

2. Socio-prophylactic approach. The main purpose of this is to identify, eliminate, neutralize the causes and conditions of negative situations. They are unaware that they are being prosecuted for drug use by minors.

They believe that drug addiction is a private affair, only that the distribution of drugs is a crime. With the passage of a new law on child drug abuse and psychotropic substances, those who use these substances will also be prosecuted.

The issue of prevention of alcoholism among children also plays an important role in our society. Children suffer from alcoholism and alcoholism nor do they know about the social and psychoneurological consequences.

Medical-biological approach. The essence of this approach should be at the biological level of treatment of various mentally ill people of medical and prophylactic nature in the prevention of deviations from social norms. If a person has mental (spiritual) development, various health defects, he can violate the spiritual and moral norms based on his mental characteristics. In this case, the person is considered irreplaceable. It is important to identify such mental illness in children in a timely manner.

Because they can perform actions that are completely unimaginable. Children should be examined by a psychiatrist and treated accordingly.

Socio-pedagogical approach. The essence of this approach is to restore or correct the personal characteristics of children in deviant behavior. In particular, it is necessary to reeducate his moral and volitional personal qualities. In children, their willpower, behavior should form the following:

\section{To have one's own word and deed;}

To stop the interest and actions of children in crimes such as crime and harm to society;

Deal with issues that are important today;

Set well-thought-out goals that are not against the law.

A person without freedom is like a flyer, the wind blows in the same direction. Such a person can be expected to violate social norms and commit crimes. It is easy to deceive him, to listen to others. it is easy to turn to other ethical views and values. By following other moral views, he acquires delinquent and immoral behavior. In educating such people, society needs to give them freedom and rights and teach them to be self-governing. Apply sanctions. The essence of this approach is to punish the offender under criminal law. 
However, the world experience shows that this is a situation that does not give Samara and is considered as a severe sanction by the society. Therefore, it is only a tool to identify and eliminate the causes of social deviation. Sociopedagogical rehabilitation work for children with deviant behavior.

The social educator's technology of working with socially disadvantaged children is to rehabilitate them.

Rehabilitation can be seen as a system of measures that integrates a person into society. Rehabilitation can also be seen as the result of an individual's influence on the functions of his or her physical psyche. Unlike adaptation (flexibility), rehabilitation is understood as the restoration of human activity. Rehabilitation is a system of measures aimed at returning a child to an active life, community and socially useful work.

Socialization is a continuous and multifaceted process. This process is especially rapid in childhood and childhood. If he imagines this process as the construction of a house, then it is in childhood that the foundation of the whole building is laid and built. Then, throughout the life, only the decoration is done. The process of socialization of the child, his formation and development as a person takes place in interaction with the environment.

There are macro (Greek macros "big"), meso (mesos "medium") and micro (micros "small") factors of a person's socialization. Microclimates - the country, society, state and secular planetary processes inhabited by man ecological, demographic, economic, sociopolitical and so on.
Mesoamyls - the formation of ethnic guidelines, the individual's perception of a particular state of national life and interethnic relations, people's views and opinions about the history of their epic (people) and modern life; the impact of regional conditions in which the child lives and develops; type of residence (city, district center, village);

Microfilms are the family, educational institutions, peer groups, etc. that make up the immediate space and social environment. this immediate environment, the environment in which the child grows, is called the social or microsocial.

Children with difficult upbringing do not follow the rules and norms established in society. This is called deviation in science (deviation in Latin). For example, in physics there is a deviation of the magnetic needle of the compass, in biology there is a deviation in the development of the organism.

Deviation is a phenomenon of change that is inherent in the person and the environment that surrounds it. Variability is related to human behavior in a social environment. As mentioned earlier, behavior can be normal or deviant.

Normative Behavior The developmental needs and opportunities of a child are his or her interaction with a micro-society that fully responds to his or her socialization. Behavior in children becomes normative if the environment around the child is always (or almost always) timely and adequate (monand) to this or that characteristic of the child, his behavior. This view also leads to a description of behavioral deviation, but the child's behavior as a social interaction that disrupts his development and socialization, as well as the moral and legal deviation established in 
society, leads to social maladaptation 'is one of the manifestations of the squeezing event).

Childhood - the following pathologies are confirmed in childhood maladaptation: Schoolage children, out-of-school children, orphans, social orphans, children addicted to drugs and poisons; sexually immoral children, children involved in delinquent activities.

Deviation includes deviant, delinquent, and criminal behavior.

Deviant behavior is a type of behavioral deviation that is associated with a violation of the norms and rules of social behavior that are not appropriate for the age of the children, microsocial relationships (family, school) and minor sexual age social groups. That is, this type of behavior can be called indiscipline. Deviant behaviors include children's protest, aggression, self-determination, systemic deviation from school and work, leaving home, drunkenness, alcoholism, alcoholism, drug addiction, and related social activities. , antisocial activities of a sexual nature, suicide.

\section{Causes of deviant behavior in children:}

Human development is based on the interaction of many factors: hereditary transmission from generation to generation, environment, upbringing, personal practical activities of man, and so on. The main factors that underlie the deviant behavior of minors can be identified.

\section{CONCLUSION}

Although the personality of the school-age student is covered by many scholars, there are some aspects of this problem that have not yet been developed in science. Therefore, in our research, we conducted our own personal research on such issues. The analysis of existing views, theories and directions on the influence of the external environment, i.e. national traditions and customs, on the formation of the behavior of school-age students, proved the urgency of this problem for the period. First, according to the results of the experiment, behavioral perceptions are the attitude to a particular type of activity, consistency, superiority, independence in their behavior.

The predominance of qualities such as responsibility, obedience, cooperation, dependence, shyness, doubt, insecurity, openness, cohesiveness. Second, the scientific theories and scientific views of the Commonwealth of Independent States and the Republic of Uzbekistan on the possibility of forming certain behavioral perceptions in human behavior have been critically studied. The weight of their scientific work in this field was determined. Third, important methodologies and methods for studying the impact of national traditions and social factors on the behavioral perception of school-age students have been clearly developed.

\section{REFERENCES}

1. Karimov IA "Independence and spirituality". «Uzbekistan», 1994, -160 p.

2. Karimov I.A. "There is no future without historical memory." Tashkent, "Sharq" 1998, -

3. Temurova S. Upbringing of children in the example of progressive traditions. Tashkent "Teacher", 1980.

4. Tokareva V.A. Aytmetova S.Sh. Methodology psychologicheskogo izucheniya shkol'nikov. Tashkent, "Teacher", 1980. 
The American Journal of Social Science and Education Innovations (ISSN - 2689-100x)

Published: December 31, 2020 | Pages: 318-330

5. http://library.ziyonet.uz/ru/book/downloa d/34050

6. https://yandex.uz/ 